\title{
The transcriptional coactivator PGC1a protects against hyperthermic stress via cooperation with the heat shock factor HSF1
}

\author{
$L X^{1,2}, X a^{1,2}, A$ Bagattin ${ }^{1}$ and $E$ Mueller ${ }^{*, 1}$
}

\begin{abstract}
Heat shock proteins (HSPs) are required for the clearance of damaged and aggregated proteins and have important roles in protein homeostasis. It has been shown that the heat shock transcription factor, HSF1, orchestrates the transcriptional induction of these stress-regulated chaperones; however, the coregulatory factors responsible for the enhancement of HSF1 function on these target genes have not been fully elucidated. Here, we demonstrate that the cold-inducible coactivator, PGC1 $\alpha$, also known for its role as a regulator of mitochondrial and peroxisomal biogenesis, thermogenesis and cytoprotection from oxidative stress, regulates the expression of HSPs in vitro and in vivo and modulates heat tolerance. Mechanistically, we show that PGC1 $\alpha$ physically interacts with HSF1 on HSP promoters and that cells and mice lacking PGC1 $\alpha$ have decreased HSPs levels and are more sensitive to thermal challenges. Taken together, our findings suggest that PGC1 $\alpha$ protects against hyperthermia by cooperating with HSF1 in the induction of a transcriptional program devoted to the cellular protection from thermal insults.
\end{abstract}

Cell Death and Disease (2016) 7, e2102; doi:10.1038/cddis.2016.22; published online 18 February 2016

Heat shock proteins (HSPs) are members of an evolutionary conserved family of proteins whose expression increases in response to a variety of different metabolic insults. ${ }^{1}$ These factors are classified primarily according to their molecular weight as HSP40, HSP60, HSP70, HSP90, HSP100 and small HSPs. ${ }^{2}$ HSP40s, also known as Dnaj proteins, are cochaperones assisting chaperones such as HSP70 and HSP90 in their functions. ${ }^{1,3}$ In stressful conditions, such as during heat shock, oxidative stresses or hypoxia, HSPs function by stabilizing unfolded or misfolded peptides, restraining protein aggregation through co-chaperone-mediated cycles of ATP hydrolysis and ADP release and by binding with chaperones until the native protein conformation is restored. When the refolding process fails, chaperones also assist the degradation of the aberrant/misfolded proteins via the ubiquitin-proteasome system. ${ }^{4}$ HSPs and their cofactors have been shown to be responsible for inhibiting both apoptotic and necrotic pathways during cell death. ${ }^{5}$ These molecular chaperones function as protectors of the proteome and have central roles in the physiology of aging and neurodegeneration. .,7 $^{6,7}$

Hyperthermia, the classic inducer of HSPs, leads to heat stroke and can arise in genetically predisposed subjects with mutations in the ryanodine receptor gene ${ }^{8}$ and in subjects with adverse reactions to drugs such as selective serotonin reuptake inhibitors, monoamine oxidase inhibitors, tricyclic antidepressants and volatile anesthetic gases. ${ }^{9}$ The family of the 70 kilodalton HSPs (HSP70s) includes 13 members in humans widely expressed within all the subcellular compartments. ${ }^{4,10}$ Among them, HSC70 (HSPA8) is a constitutively expressed chaperone, while HSPA5 (GRP78), localized to the endoplasmic reticulum, and HSPA9 (mtHSP70), present in the mitochondrial compartment, are stress-induced. HSP70 paralogs HSPA1A, HSPA1B and HSPA1L have been shown to be involved in the refolding of damaged or defective proteins, in their targeting for degradation and for directly inhibiting apoptosis, thus ultimately protecting cells from thermal and oxidative stress. ${ }^{10}$

The transcriptional activation of the heat shock response is orchestrated by the heat shock factor 1 (HSF1). ${ }^{1,11}$ HSF1 exists as an inactive monomer in a complex with Hsp40, $\mathrm{Hsp} 70$ and Hsp90. Upon physiological stresses resulting from hyperthemia or oxidative insults, HSF1 is released from the chaperone complex and, upon trimerization, it translocates into the nucleus and binds to heat shock-responsive elements (HSEs) present on target gene promoters to initiate their transcriptional activation. ${ }^{12}$ It has been shown that HSF1-null fibroblasts are more susceptible to heat-induced apoptosis because of the absence of HSP induction ${ }^{13}$ and that HSF1-null mice fail to raise HSP levels in response to thermal insults, ${ }^{14}$ suggesting critical roles of HSF1 in the regulation of HSPs in stress defense. In addition to the transition of active and inactive states of HSF1 by association with HSPs, previous studies have also reported that the activation and attenuation of HSF1 activity is achieved through extensive posttranslational modifications, including phosphorylation, sumoylation and acetylation. ${ }^{12}$ Although HSF1 has been

\footnotetext{
${ }^{1}$ Genetics Development and Disease Branch, National Institute of Diabetes, Digestive and Kidney Diseases, National Institutes of Health, Bethesda, MD, USA *Corresponding author: E Mueller, Genetics Development and Disease Branch, National Institute of Diabetes, Digestive and Kidney Diseases, National Institutes of Health, 9000 Rockville Pike, Building 10, Room 8D12, Bethesda, MD 20814, USA. Tel: +1 301594 6797; Fax: +1 301402 4136; E-mail: elisabettam @mail.nih.gov ${ }^{2}$ These authors contributed equally to this work.

Abbreviations: HSP, heat shock protein; ATP, adenosine triphosphate; ADP, adenosine diphosphate; HSE, heat shock element; ROS, reactive oxygen species; MPTP, (1-methyl-4-phenyl-1,2,3,6-tetrahydropyridine); S.E.M., standard error of the mean; DMEM, Dulbecco's modified Eagle's medium; FBS, fetal bovine serum; GFP, green fluorescent protein

Received 07.10.15; revised 14.1.16; accepted 19.1.16; Edited by M Agostini
} 
implicated in counteracting cell stress and is critical in cancer biology, aging and neurodegenerative diseases, little is known about the regulatory molecules and complexes that modulate its activity. ${ }^{1,15}$

Peroxisome proliferator-activated receptor $\gamma$ coactivator $1 a$ (PGC1a) is a cold-inducible coactivator that controls mitochondrial and peroxisome biogenesis and energy metabolism. ${ }^{16,17}$ In addition to these functions, it has been shown that PGC1 $a$ has dual roles in maintaining cellular and tissue homeostasis by increasing mitochondrial activity and preventing oxidative stress through the induction of reactive oxygen species (ROS)-detoxifying enzymes. ${ }^{18}$ Studies in wildtype (WT) and PGC1a-null mice have demonstrated that absence of PGC1a in vivo is associated with striatum lesions and increased dopaminergic cell death and oxidative damages upon treatment with MPTP, a compound known to induce neourotoxicity in animal models and humans. ${ }^{18,19}$ Despite the clear role of PGC1a in ROS protection, less is known about the function of PGC1 1 in response to other types of stressors. Recently, the results of genome-wide sequencing of PGC1a-binding sites in hepatocytes have suggested the potential occupancy of PGC1a at the heat shock elements (HSE) present at promoters of HSPs. ${ }^{20}$ In addition, recent data from our laboratory have demonstrated the physical interaction and co-occupancy of PGC1a-HSF1 at the HSE present on the promoter of PGC1a. ${ }^{21}$ On the basis of these reports, we hypothesized the potential cooperation of PGC1 $a$ and HSF1 in regulating $\mathrm{HSPS}$ under stress conditions and assessed the possible role of PGC1a in the protection from hyperthermic stress and its pathological consequences in animals.

Here, we report that PGC1a is sufficient to induce HSPs both in vitro and in vivo and that it interacts with HSF1 at the HSE present at the promoter of HSP70 after heat shock stimuli. Furthermore, we show that cells with deficiency in PGC1 $a$ have reduced HSPs levels and that are more sensitive to hyperthermia-induced apoptosis. In addition, PGC1a knockout (KO) mice show decreased ability to adapt to changes in temperatures as demonstrated by the increased apoptosis in kidney and liver after thermal challenge. Restoration of PGC1a levels in PGC1a KO cells and livers rescued heat tolerance. Overall, our results establish PGC1a as a new critical orchestrator of thermotolerance in cooperation with HSF1.

\section{Results}

PGC1a is sufficient to induce HSPs in vitro. Given our recent data demonstrating the existence of a transcriptional cooperation between PGC1a and HSF1 in the context of energy metabolism ${ }^{21}$ and the previously reported function of PGC1a in protection from stress, ${ }^{18}$ we hypothesized that PGC1a may have a role in cellular defense against heat shock through its cross talk with HSF1. To assess this, we first systematically analyzed the expressions of a number of genes known to be involved in heat shock responses in cells with gain- or loss-of-function of PGC1a. This analysis revealed that PGC1a ectopic expression in 10T1/2 cells significantly induced $32 \%$ of the genes commonly known to be involved in heat shock responses (Figure 1a and
Supplementary Table 1). Conversely, PGC1a ablation in fibroblasts was associated with a reduction in the levels of $29 \%$ of these HSPs and chaperone genes when cells were exposed to heat shock for $1 \mathrm{~h}$ (Figure $1 \mathrm{~b}$ and Supplementary Table 2). Cross comparison analysis of genes modulated both in cells with upregulation or downregulation of PGC1a revealed that 15 genes, including 5 HSP40s (Dnaja3, Dnaja4, Dnajb2, Dnajc16 and Dnajc19), 1 HSP60 (Hspd1), 3 HSP70s (Hspa1a, Hspa1b and Hspa9), 2 small HSPs family members (Hspb7/Hsp27 and Serpinh1/Hsp47) and 4 other chaperones (Bag3, Cct4, Cryab and Pfdn1) are possible targets of PGC1a given that their expression levels are dependent on PGC1a (Figure 1c). The increased levels of representative HSPs of different categories, such as Dnaj3, Dnajc19, HSPd1, HSPa1a, HSPa9 and Bag3, were further validated by real-time PCR in 10T1/2 cells overexpressing PGC1a (Figure 1d).

It has been previously demonstrated that PGC1 $a$ is a stressinduced protein, activated by a number of challenges, including ROS. ${ }^{18}$ We therefore next examined whether the mRNA levels of HSPs were regulated under the same conditions known to induce PGC1a and genes involved in ROS responses. As shown in Figure 1e, HSPs mRNAs were elevated following exposure to hydrogen peroxide, with UCP2 expression levels used as a positive control. These results demonstrate that PGC1a is both sufficient and necessary to induce the expression of HSPs in vitro in response to cellular stressors.

PGC1a modulates HSP levels in vivo. We next examined whether PGC1a regulates HSPs also in vivo. Given that the expression of PGC1a is cold-inducible, ${ }^{22}$ we determined whether HSPs would be induced in mice exposed to $4^{\circ} \mathrm{C}$. As shown in Figures $2 a$ and $b$, we observed an induction of HSPs in brown and inguinal fat in the same conditions that elicited an increase in the expression of PGC1a and UCP1 in these tissues, suggesting that the levels of these HSPs are regulated by hypothermia. Interestingly, the acute exposure to cold did not elevate the levels of HSPs in tissues such as heart and kidney in which PGC1a mRNAs are not regulated in response to low temperatures (Supplementary Figures 1a and b) nor damaged liver and kidney as indicated by the absence of apoptotic events in these two tissues (Supplementary Figure 1c).

To further examine whether gain of PGC1a function would be associated with the expression of HSP in vivo, we took advantage of adenoviral delivery of PGC1a in tissues by following the same technical procedures that we previously used to successfully achieve expression of specific genes in selected depots. ${ }^{21,23}$ This analysis revealed that HSPs are induced in inguinal fat (iWAT) overexpressing PGC1a by adenoviral injection but not in control-injected iWAT (Figure 2c). Of note, these adenoviral injections did not alter the levels of PGC1 a nor caused any morphological changes in organs such as brown adipose tissue (BAT), liver, kidney and heart (Supplementary Figures 1d and e).

PGC1a is required for the HSF1-dependent induction of Hsp70 in hyperthermia. In order to investigate whether PGC1a is indispensable for the induction of HSPs in 
a

10T1/2

Ad-GFP Ad-PGC1a

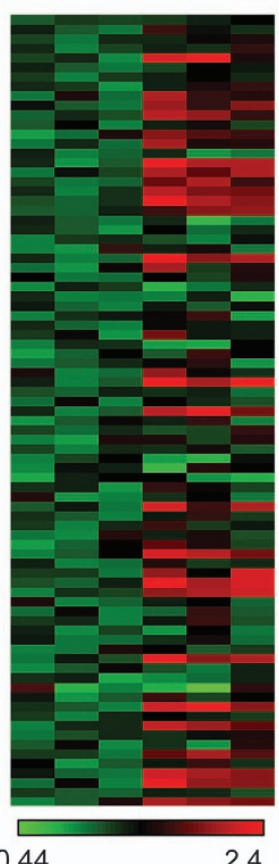

0.44

\section{b}

Fibroblasts after HS

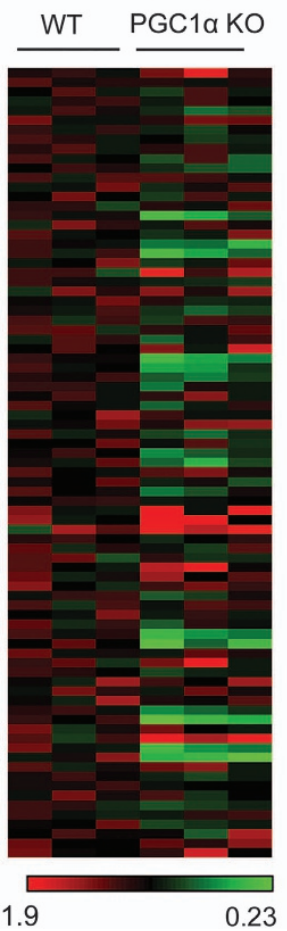

C

10T1/2

Froblasts after HS

\section{Ad-GFP Ad-PGC1a}

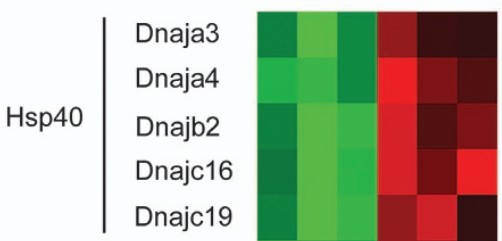

Hsp60

Dnajc19

Hspa1a

Hsp70

Hspa1b

Hspa9

Small Hsps

$\mathrm{Hspb} 7$

Serpinh1

Bag3

Cct4

Others

Cryab

Pfdn1

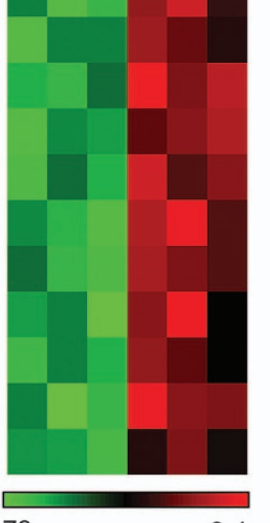

0.78

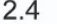

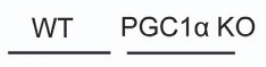

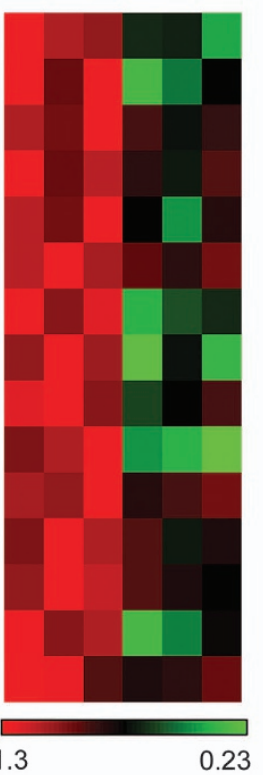

0.23

d
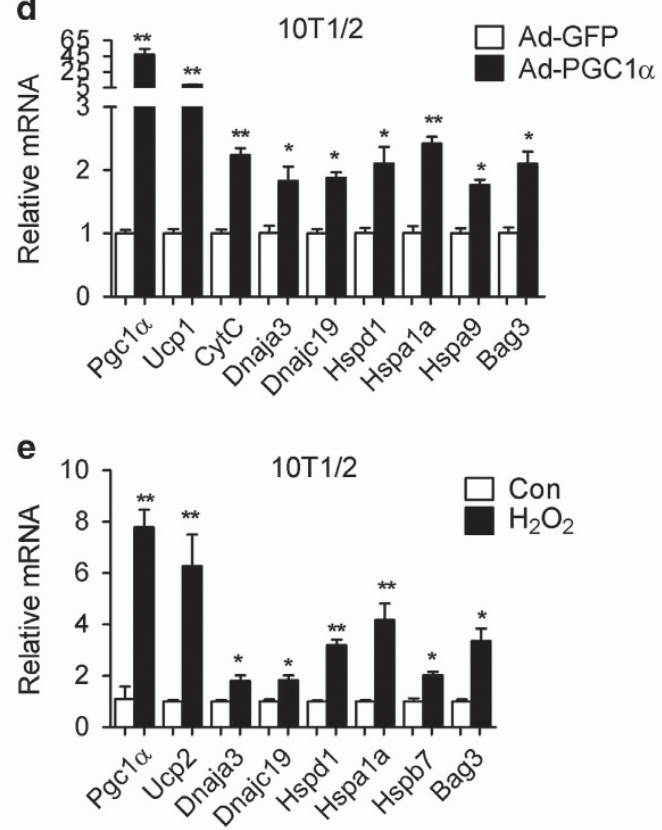

Figure $1 \mathrm{PGC1} \alpha$ is sufficient to induce the expression of HSPs in vitro. (a-c) Heat map representation of 84 differentially regulated HSP genes (red, high; green, low) identified by PCR array analysis in 10T1/2 cells infected with control or PGC1 $\alpha$ adenovirus for 3 days (a) and in WT and PGC1 $\alpha$-null fibroblasts exposed to heat shock for $1 \mathrm{~h}$ at $42{ }^{\circ} \mathrm{C}$ or at $37^{\circ} \mathrm{C}$ (b) and of 15 common regulated HSP genes from two arrays (c). (d) mRNA levels of PGC1 $\alpha$, UCP1, CytC and HSPs in $10 T 1 / 2$ cells infected with control or PGC1 $\alpha$ adenovirus for 3 days. (e) mRNA levels of PGC1 $\alpha$, UCP2 and HSPs in 10T1/2 cells treated with $\mathrm{H}_{2} \mathrm{O}$ or $0.5 \mu \mathrm{M}$ hydrogen peroxide for $2 \mathrm{~h}$ and subsequently allowed to recover for $2 \mathrm{~h}$. Error bars represent S.E.M. and data are presented as mean \pm S.E.M. ${ }^{*} P<0.05,{ }^{*} P<0.01$ compared with controls 

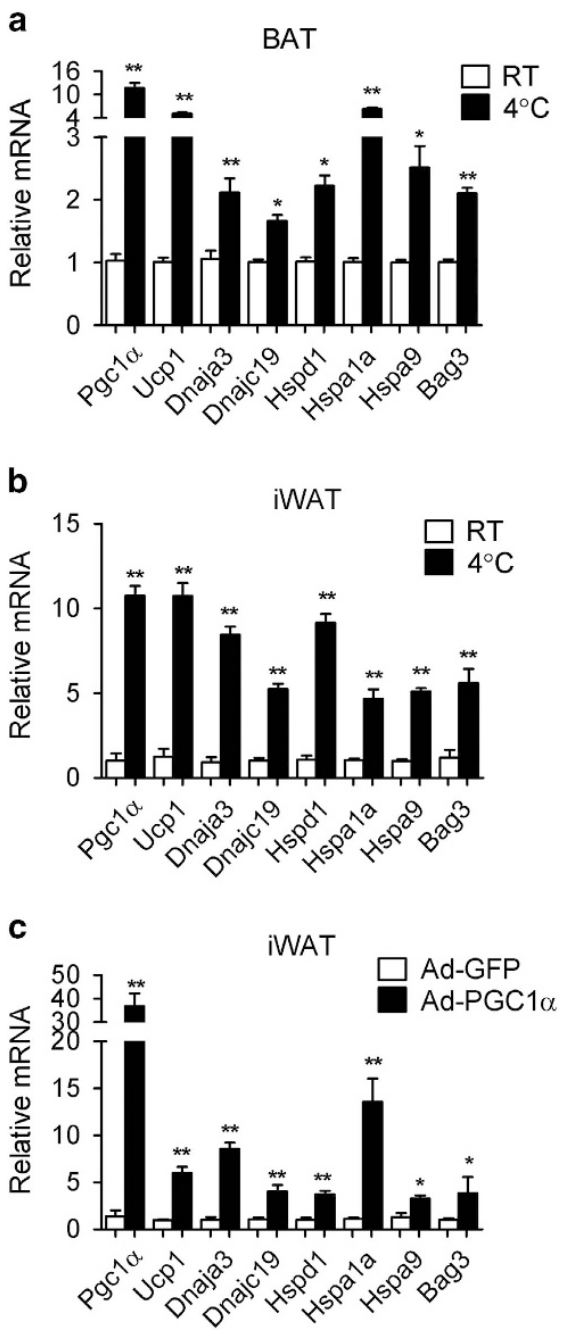

Figure $2 \mathrm{PGC1} \alpha$ modulates HSP expression in vivo. (a and $\mathbf{b}$ ) mRNA levels of PGC1 $\alpha$, UCP1 and HSPs in BAT (a) and iWAT (b) of mice kept at room temperature or after cold exposure for $4 \mathrm{~h}$. (c) mRNA levels of PGC1 $\alpha$, UCP1 and HSPs in iWAT injected with control or PGC1 $\alpha$-expressing adenovirus. Error bars represent S.E.M. and data are presented as mean \pm S.E.M. ${ }^{\star} P<0.05,{ }^{* \star} P<0.01$ compared with controls. $n=4$ per group

response to hyperthermia, we subjected WT and PGC1a-null cells to acute heat shock and analyzed the modulation of Hspa1a/Hsp70 levels given that this gene is among the most well-studied stress-inducible Hsp70 of all HSPs and its transcriptional regulation by HSF1 in response to heat shock is well documented. This analysis revealed that whereas Hspa1a/Hsp70 mRNA was induced in WT cells, as expected, its levels were blunted in PGC1a-null cells (Figure 3a and Supplementary Table 2). Conversely, PGC1a overexpression enhanced Hspa1a/Hsp70 levels after heat shock (Figure 3b). Given that the expression of HSPs is regulated by HSF1, we analyzed the effects of PGC1a overexpression in HSF1-deficient cells. As shown in Figure $3 c$ and Supplementary Figure 2, ectopic expression of PGC1a was not sufficient to increase Hspa1a/Hsp70 levels in the absence of HSF1, suggesting that PGC1a requires the transcription
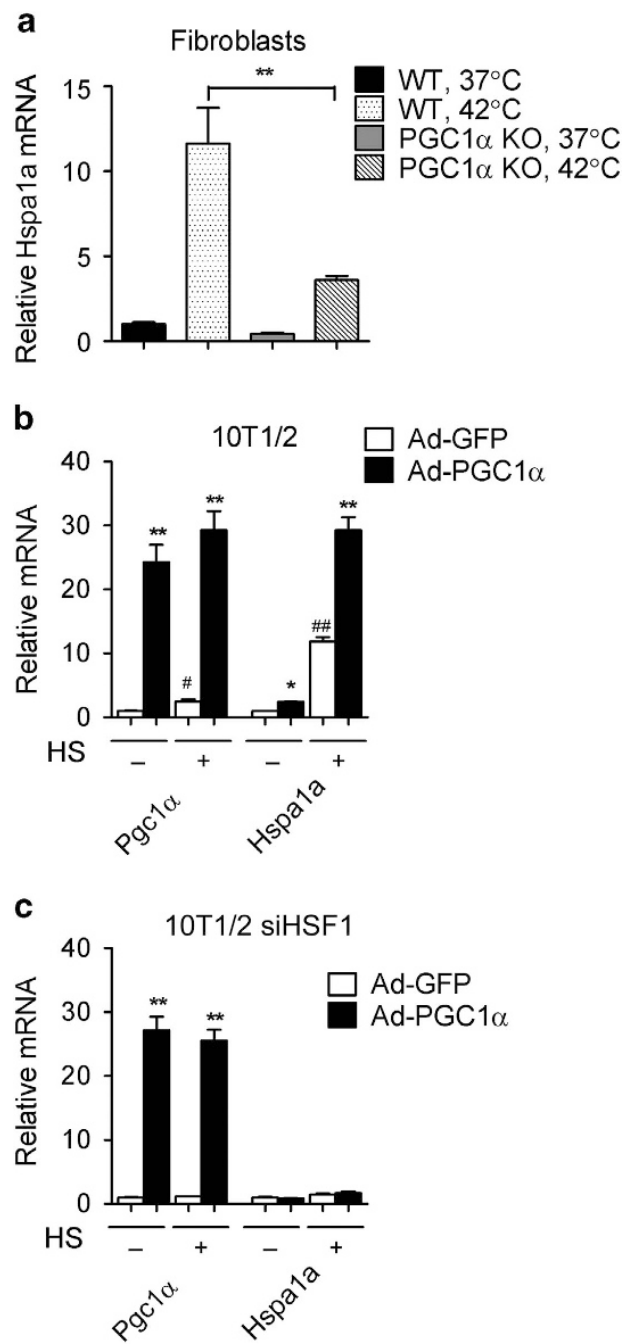

Figure $3 \mathrm{PGC1} \alpha$ is required for HSF1-dependent induction of the HSP70 family member Hspa1a. (a) mRNA levels of Hspa1a in WT and PGC1 $\alpha$-null fibroblasts exposed to heat shock $\left(42^{\circ} \mathrm{C}\right)$ for $1 \mathrm{~h}$ or left at $37^{\circ} \mathrm{C}$. (b) mRNA levels of Hspa1a in $10 \mathrm{~T} 1 / 2$ cells infected with adenovirus expressing control or PGC1 $\alpha$ and treated after $1 \mathrm{~h}$ of heat shock at $42{ }^{\circ} \mathrm{C}$ or at $37^{\circ} \mathrm{C} .{ }^{\#} P<0.05,{ }^{\#} P<0.01$, comparison of Ad-GFP group with or without heat shock (HS) treatment. (c) mRNA levels of Hspa1a in $10 T 1 / 2$ cells with HSF 1 knockdown, infected with either control or PGC1 $\alpha$ adenovirus and subjected to heat shock at $42^{\circ} \mathrm{C}$ for $1 \mathrm{~h}$ or left at $37^{\circ} \mathrm{C}$. Error bars represent S.E.M. and data are presented as mean \pm S.E.M. ${ }^{*} P<0.05,{ }^{* \star} P<0.01$ compared with controls

factor HSF1 to modulate the expression of this member of the Hsp70 family.

PGC1a and HSF1 physically interact and functionally cooperate to transcriptionally activate Hsp70. Given the requirements of PGC1a for the induction of the HSF1 target genes, we sought to determine the mechanisms through which PGC1a affects Hspa1a/Hsp70 mRNA levels. Transcriptional assays revealed that gain of PGC1a function led to the induction of luciferase driven by a HSE in a dosedependent manner and that the coexpression of PGC1 $a$ and HSF1 further increased the activity of this reporter gene (Figure 4a), suggesting that PGC1a and HSF1 synergistically 
a

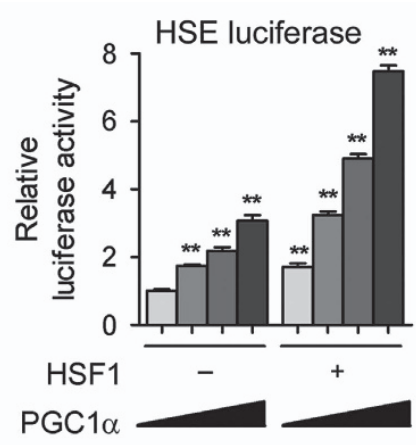

b
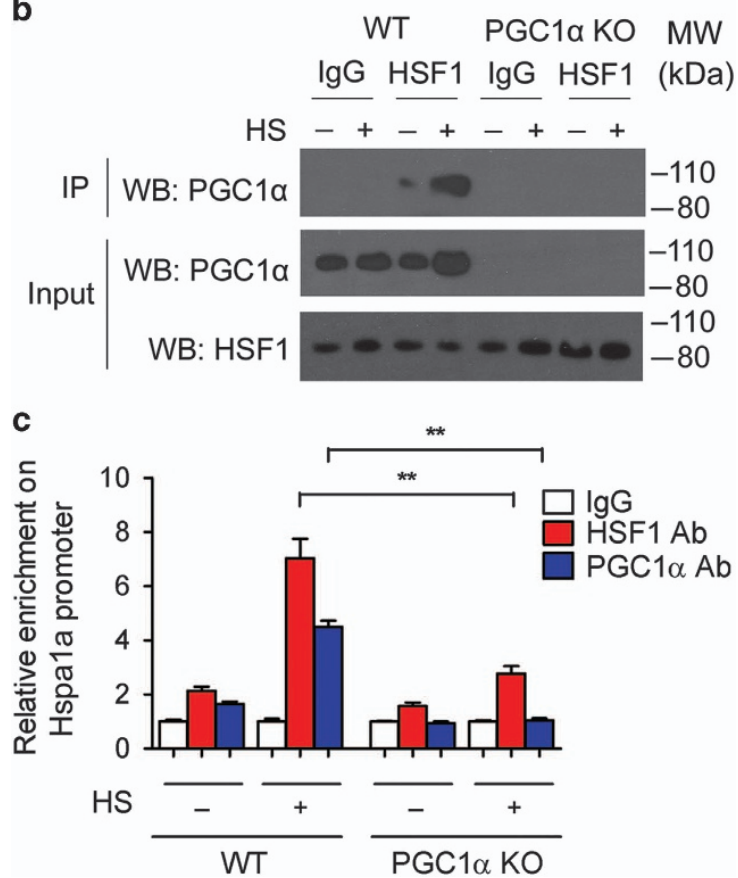

Figure $4 \mathrm{PGC1} \alpha$ and HSF1 physically interact and functionally cooperate on heat shock response elements. (a) Luciferase assay performed on a reporter containing a HSE in 10T1/2 cells co-transfected with PGC1 $\alpha$, HSF1 and vector controls. (b) Co-IP assay to assess the interaction between PGC1 $\alpha$ and HSF1 in WT and PGC1 $\alpha$-null brown adipocytes exposed to heat shock at $42{ }^{\circ} \mathrm{C}$ (HS) for $1 \mathrm{~h}$ and recovered for an additional hour. (c) ChIP assay at the Hsp70 promoter after $1 \mathrm{~h} 42{ }^{\circ} \mathrm{C}$ heat shock and $1 \mathrm{~h}$ recovery in WT and PGC1 $\alpha$-null brown adipocytes to assess the binding of PGC1 $\alpha$ and HSF1. Error bars represent S.E.M. and data are presented as mean \pm S.E.M. ${ }^{*} P<0.05,{ }^{* \star} P<0.01$ compared with controls

activate genes controlled by HSE. Furthermore, our analysis revealed that PGC1 $a$ and HSF1 physically interact and that their binding is enhanced during heat shock (Figure 4b). Through ChIP assay, we confirmed that PGC1a and HSF1 bind to the HSE element present at the Hsp70 promoter after heat shock. Conversely, we observed reduced binding of HSF1 to the HSE element present on the promoter of Hsp70 in PGC1a-null cells (Figure 4c and Supplementary Figure 3). Together, these data indicate that PGC1a interacts with HSF1 and cooperates in the transcriptional activation of Hsp70.

PGC1a-deficient cells are sensitive to heat stress. Given our results indicating impaired induction of HSPs after hyperthermic stress in cells lacking PGC1a (Supplementary
Table 2 and Figure $3 a$ ), we tested whether PGC1a is necessary for cellular protection from hyperthermia. As shown in Figures 5a-c, when PGC1a-null cells were exposed to heat shock, they showed reduced viability (Figure 5a) and increased apoptosis in comparison with WT cells (Figures 5b and c). Consistently, we observed increased cleavage of caspase-3 in PGC1a-null cells exposed to heat shock (Figure 5d). Complementation studies demonstrated that restoration of PGC1a levels in PGC1a-null cells by adenovirus was associated with elevation of HSP levels (Figure 5e) and increased resistance to apoptosis in response to heat stress (Figure 5f). These data indicate that PGC1 $a$ has critical and unexpected roles in regulating cellular responses to hyperthermia.

PGC1a is required for heat resistance in response to thermal challenge in mice. In order to characterize the in vivo significance of PGC1a function during hyperthermic stress, we exposed WT and PGC1a-null mice to sub-lethal temperatures. Analysis of organs including BAT, iWAT, gastrocnemius, kidney, liver, spleen and brain revealed an elevation of Hspa1a/Hsp70 levels after heat shock in WT mice compared with mice lacking PGC1a, suggesting that $\mathrm{PGC1a}$ is required for Hsp70 induction after hyperthermia in vivo (Figure 6a). Furthermore, we observed an increase in PGC1a levels in parallel to those of Hsp70 in response to heat, supporting the possibility that HSF1 induces PGC1a and that requires it for HSP induction (Supplementary Figure 4a). Given the evidence that PGC1a can protect against apoptosis induced by hyperthermia in vitro (Figure 5) and that liver and kidney are the primary loci affected during heat stroke in humans, ${ }^{24}$ we analyzed WT and PGC1a KO mice and showed increased apoptosis, increased cytosolic cytochrome c (CytC) release from mitochondria and elevated caspase-3 activity in these two organs in PGC1a-null mice after heat shock (Figures $6 b-f)$. In addition, restoration of PGC1a levels in liver of PGC1a-null mice by tail vein adenoviral injections was associated with increased Hspa1a mRNA levels (Figure $6 \mathrm{~g}$ ) and enhanced resistance to apoptosis after heat shock (Figure 6h). These results suggest that absence of PGC1a is associated with reduced levels of HSPs and impaired responses to thermal challenges.

\section{Discussion}

The analyses performed here demonstrate that PGC1a can cooperate with HSF1 in the induction of a number of HSPs in a variety of tissues and cells in response to different stimuli, such as thermal and oxidative stress. Furthermore, loss-of-function studies showed that PGC1a is required for HSF1 activation of HSPs and protection from hyperthermia. This cytoprotective role of PGC1a exerted through the activation of HSPs is consistent with the previously characterized function of this coactivator in cell defense in response to a variety of insults, as reported in the context of transcriptional activation of detoxifying genes in cells exposed to $\mathrm{ROS}^{18}$ and in the unfolded protein response regulated in cooperation with ATF6. ${ }^{25}$ It is plausible that PGC1a may complex with other transcription factors in addition to HSF1 to induce stress-induced proteins; therefore, future studies involving mass spectrometry and 
a

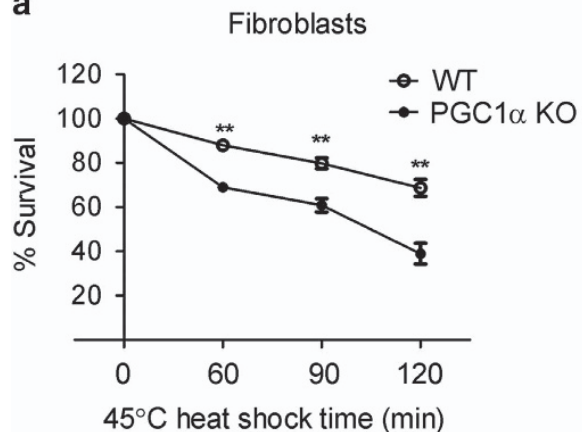

c

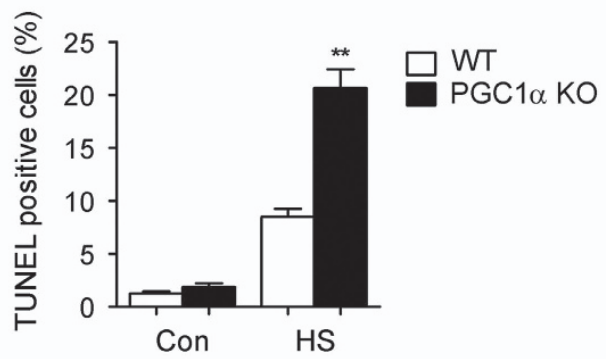

b

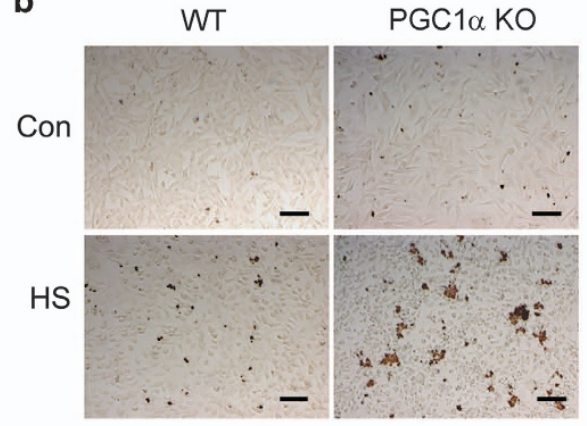

d
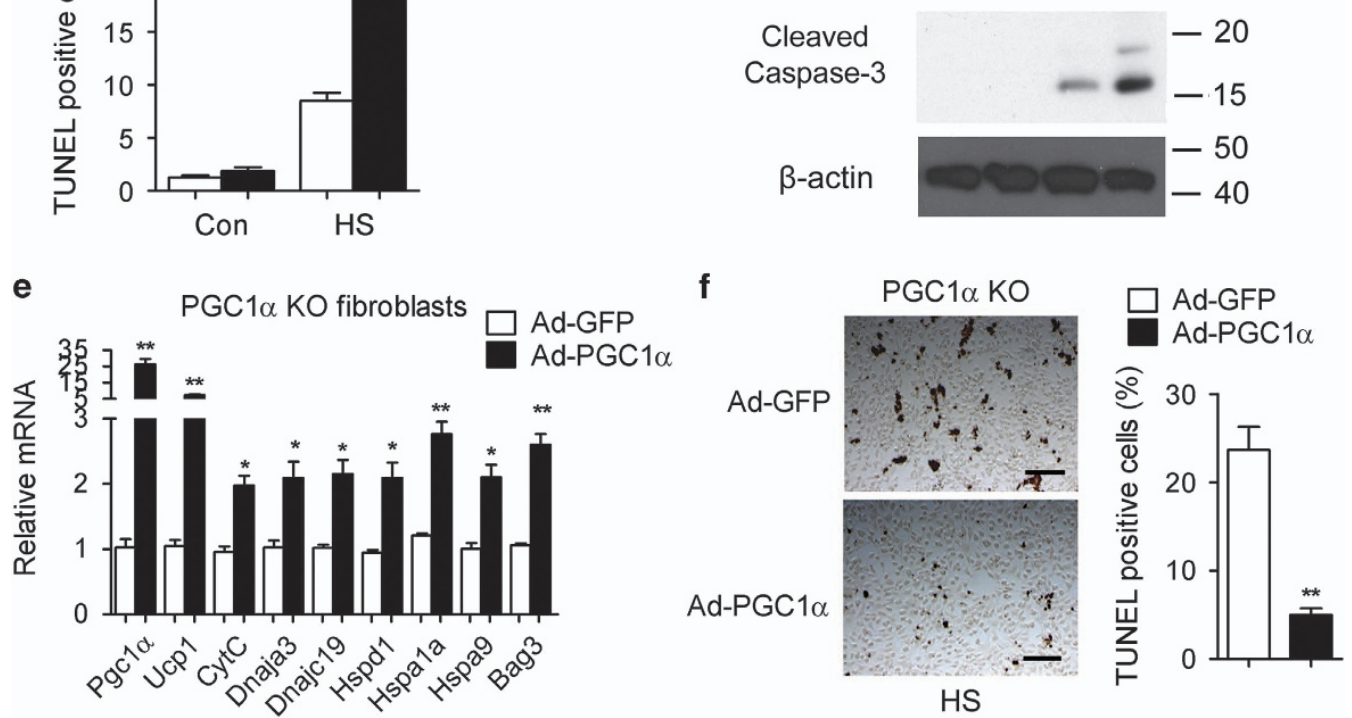

Figure 5 PGC1 $\alpha$-deficient cells are less resistant to heat stress. (a) Survival curve of WTand PGC1 $\alpha$-null fibroblasts after heat shock at $45^{\circ} \mathrm{C}$ for the indicated time. (b and c) Representative images of TUNEL staining (b) and quantification of TUNEL-positive cells (c) in WT and PGC1 $\alpha$-null cells kept at $37^{\circ} \mathrm{C}$ or exposed to heat shock at $43^{\circ} \mathrm{C}$ for $1 \mathrm{~h}$ and recovered for $30 \mathrm{~min}$ at $37^{\circ} \mathrm{C}$. (d) Protein levels of cleaved Caspase-3 in WTand PGC1 $\alpha$-null cells after exposure to heat shock at $43^{\circ} \mathrm{C}$ for $1 \mathrm{~h}$ and recovered for 30 min at $37^{\circ} \mathrm{C}$ or in control cells kept at $37^{\circ} \mathrm{C}$. (e) mRNA levels of HSPs in PGC1 $\alpha$-null cells expressing control or complemented with PGC1 $\alpha$. (f) Representative images of TUNEL staining and quantification of TUNEL-positive cells in PGC1 $\alpha$-null cells either with control or re-expressing PGC1 $\alpha$ after heat shock at $43^{\circ} \mathrm{C}$ for $1 \mathrm{~h}$ and recovery for 30 min at $37^{\circ} \mathrm{C}$. Scale bar represents $100 \mu \mathrm{m}$. Error bars represent S.E.M. and data are presented as mean \pm S.E.M. ${ }^{*} P<0.05,{ }^{* *} P<0.01$ compared with controls

ChIP-seq analysis will be instructive in defining which tissueselective transcriptional factors cooperate with PGC1a to coordinate organ-selective responses to distinct stressors.

Our analysis revealed for the first time that PGC1a is necessary for the induction of Hspa1a/Hsp70/Hsp72. Given the evidence provided by previous studies demonstrating that this HSP is induced during cold exposure ${ }^{26}$ and via gain- and loss-of-function analyses showing its requirements for cold resistance in vivo, ${ }^{27}$ it can be postulated that Hspa1a/Hsp70/ Hsp72 may be an essential target of PGC1a for the expletion of its thermogenic function. In addition to its role in protecting from oxidative stress, this Hsp has also been shown to be involved in the control of insulin resistance through its negative effects on JNK phosporylation. ${ }^{28}$ Given the previously recognized role of PGC1a in energy expenditure and insulin sensitivity, it is conceivable that Hspa1a/Hsp70/Hsp72 may mediate other metabolic beneficial effects elicited by PGC1a, in addition to its function in cytoprotection in response to hyperthermic stresses.

It has been previously demonstrated that PGC1a is involved in organelle remodeling and biogenesis. ${ }^{16,17}$ The data reported here suggest that mitochondrial HSP proteins such as Hspd1/HSP60 and Hspa9/mtHSP70 devoted to organelle protection from proteotoxic stress are regulated by PGC1a. Consistently with our findings, Rera et al. ${ }^{29}$ demonstrated that drosophila overexpressing PGC1 $a$ have increased HSP60 levels in both whole larvae and adult thoraxes and increased mitochondrial activity. Given that these mitochondrial HSPs have been postulated to be under the transcriptional control of $\mathrm{HSF} 1,{ }^{30}$ our results provide novel insights into the broader function of PGC1 $a$ and its transcriptional partners in the regulation of mitochondrial biology.

Genetic mouse models have demonstrated that ablation of HSPs such as HSP70 and HSP60 can cause neurological 
a

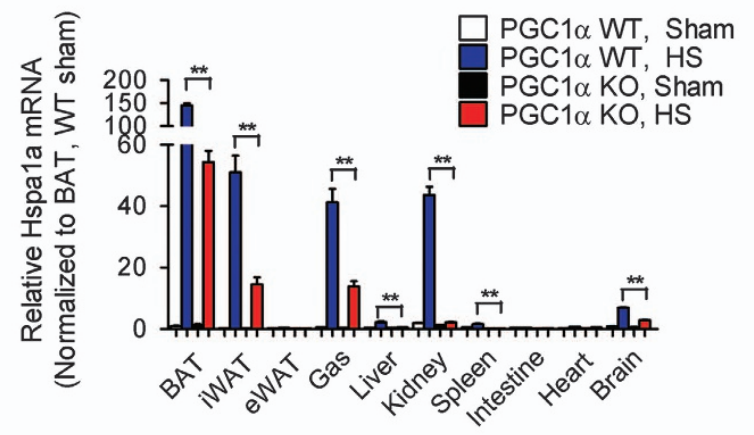

b

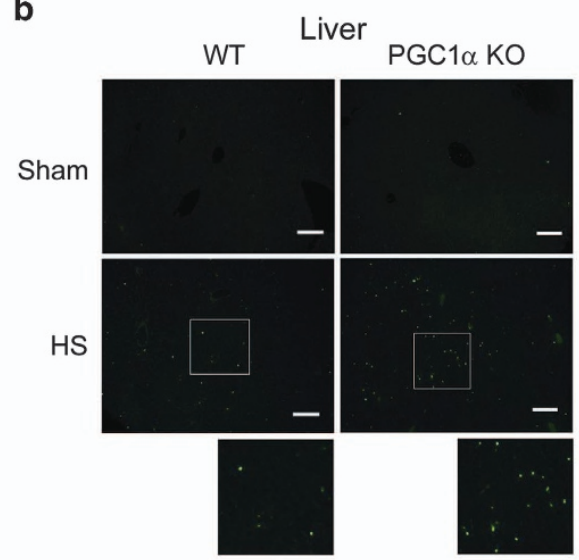

e
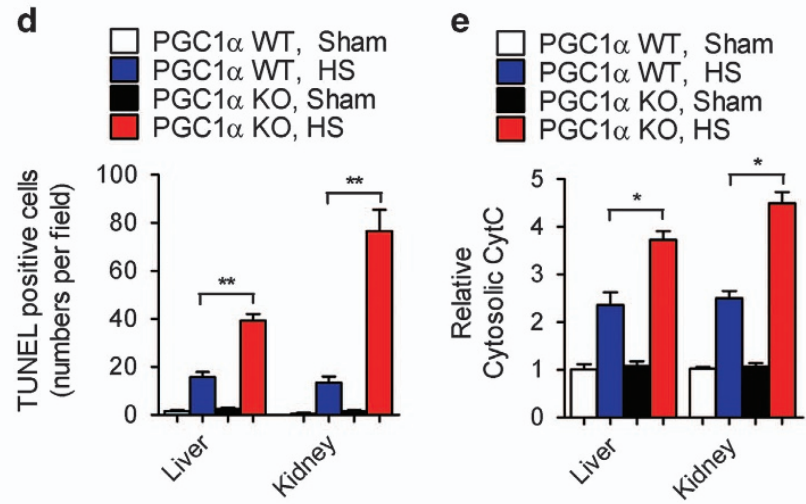

h f
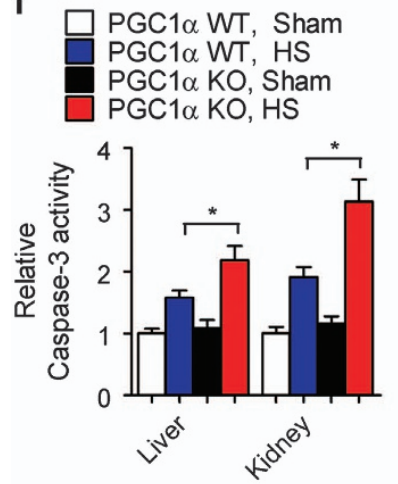

g

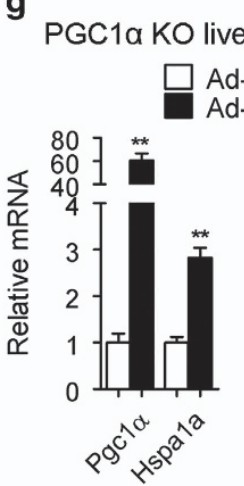

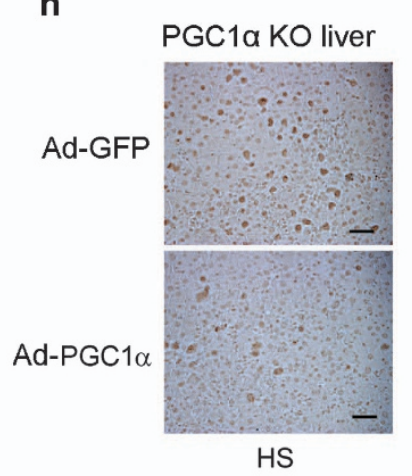

Figure 6 PGC1 $\alpha$-null mice exhibit impaired responses to thermal challenges. (a) mRNA levels of Hspa1a in tissues of WT and PGC1 $\alpha$-null mice exposed to heat shock or kept at room temperature (RT). (b and $\mathbf{c}$ ) Representative images of TUNEL staining in liver and kidney from WTand PGC1 $\alpha$-null mice after heat shock or at RT. Images from heat shock groups were magnified digitally (below) to show details of the staining. Scale bar represents $100 \mu \mathrm{m}$. (d) Quantification of numbers of TUNEL-positive cells per field in liver and kidney sections from WT and PGC1 $\alpha$-null mice exposed to heat shock or kept at RT. (e and f) Relative levels of released cytochrome c in cytosol and Caspase-3 activity in liver and kidney from WT and PGC1 $\alpha$-null mice exposed to heat shock or kept at RT. (g) mRNA level of Pgc1 $\alpha$ and Hspa1a in livers from PGC1 $\alpha$-null mice either infected with control or PGC1 $\alpha$ through tail vain injection of adenovirus. $n=3$ per group. (h) Representative images of TUNEL staining and quantification of TUNEL-positive cells in livers from PGC1 $\alpha$-null mice either with control or re-expressing PGC1 $\alpha$ after heat shock. Scale bar represents $200 \mu \mathrm{m} . n=3$ per group. Error bars represent S.E.M. and data are presented as mean \pm S.E.M. ${ }^{\star} P<0.05,{ }^{\star *} P<0.01$ compared with controls. $n=5$ per group

defects including cerebral ischemia, Huntington's disease and motor neuron disorders. ${ }^{31-33}$ Given that it has been previously shown that ablation of PGC1a in vivo is associated with brain abnormalities, ${ }^{18,19}$ it is possible that part of the PGC1a-null mice phenotype may be due to decreased levels of HSPs in this organ. Taken together, our study demonstrates for the first time that $\mathrm{PGC1} 1 \mathrm{a}$ regulates $\mathrm{HSPs}$ and protects against hyperthermic stress in cooperation with HSF1, indicating a critical novel mechanism of cellular protection exerted by PGC1 $a$ and suggesting a broader role of PGC1a in cellular defense in response to a variety of insults.

\section{Materials and Methods}

Cell culture. 10T1/2 cells were maintained in DMEM supplemented with $10 \%$ fetal bovine serum (FBS). For proteotoxic stress treatment, 10T1/2 cells were treated with hydrogen peroxide (Sigma, 216763, St. Louis, MO, USA). Immortalized 
WT and PGC1 $\alpha$-null mouse fibroblasts, a generous gift of Bruce Spiegelman (Harvard Medical School, Boston, MA, USA), were maintained in DMEM supplemented with 10\% FBS, $20 \mathrm{nM}$ insulin and $1 \mathrm{nM}$ T3 (maintenance medium). Differentiation was induced by treating confluent cells with $0.5 \mathrm{mM}$ isobutylmethylxanthine, $125 \mu \mathrm{M}$ indomethacin and $0.5 \mu \mathrm{M}$ dexamethasone for 2 days. For adenoviral experiments, $10 T 1 / 2$ cells, WT or PGC1 $\alpha$-null fibroblasts were infected with $600 \mathrm{MOI}$ of adenovirus (control and PGC1 $\alpha$ adenoviruses were constructed, amplified and purified by Vector Biolabs, Malvern, PA, USA) for $2 \mathrm{~h}$ in $0.5 \%$ FBS medium and subsequently cultured in $10 \%$ FBS medium. Ten percent FBScontaining medium was changed after $24 \mathrm{~h}$ and gene expression was determined $72 \mathrm{~h}$ after adenovirus infection, in cells exposed to heat shock or $37^{\circ} \mathrm{C}$ as control in a water bath placed in the incubator. For viability measurements, cells were stained with trypan blue after exposure to $45^{\circ} \mathrm{C}$ for the indicated time and the percentage of live cells was quantified using a haemocytometer.

Plasmids and reagents. The HSE luciferase reporter vector was purchased from Affymetrix Panomics (LR0038, Affymetrix, Santa Clara, CA, USA). The PGC1 $\alpha$ and HSF1-expressing plasmids were kind gifts of Bruce Spiegelman (Harvard Medical School) and Carl Wu (National Cancer Institute), respectively. For luciferase assay, $10 T 1 / 2$ cells plated in 24 wells were transfected with $0.1 \mu \mathrm{g}$ of HSE luciferase reporter, $0,25,50$ or $100 \mathrm{ng}$ of PGC1 $\alpha$ plasmids in the presence or absence of $10 \mathrm{ng}$ of HSF1 using a Nucleofector 96-well system (Amaxa, Lonza, Basel, Switzerland), according to the manufacturer's instructions. Control plasmid was used to even the total amount of DNA transfected in each well. Luciferase activity was assayed $48 \mathrm{~h}$ after transfection, according to protocols provided by the manufacturer (Promega, Fitchburg, WI, USA) using VICTOR3V (PerkinEImer, Waltham, MA, USA).

RNA analysis and PCR arrays. Total RNA was extracted from cultured cells or tissues with TRIzol (Invitrogen, Carlsbad, CA, USA) and $1 \mu \mathrm{g}$ total RNA was retrotranscribed into CDNA with First Strand CDNA Synthesis Kit (Roche, Basel, Switzerland), according to the manufacturers' instructions. Quantitative real-time PCR was performed using with the ABI PRISM 7900HT sequence detection system (Applied Biosystems, Thermo Scientific, Waltham, MA, USA) using SYBR green (Roche). Gene expression levels were determined by the delta delta $\mathrm{Ct}$ method, normalized to $36 \mathrm{~B} 4$ expression levels. The sequence of primers used for real-time PCR is reported in Supplementary Table 3. One microgram of total RNA from 10T1/2 cells infected with Ad-control or Ad-PGC1 $\alpha$ or WT and PGC1 $\alpha$ null fibroblasts after $1 \mathrm{~h}$ heat shock was retrotranscribed into $C D N A$ and used to perform Mouse Heat Shock Proteins \& Chaperones PCR arrays (PAMM-076Z, Qiagen, Hilden, Germany) according to the manufacturer's instructions.

Animal experiments. All animal experiments were performed according to guidelines of the National Institute of Diabetes and Digestive and Kidney Diseases Animal Care and Use Committee. PGC1 $\alpha$-heterozygous mice were a generous gift from BM Spiegelman (Harvard Medical School). To test gene expressions and apoptotic events after cold exposure, mice were individually caged and exposed to $4{ }^{\circ} \mathrm{C}$ or maintained at room temperature with free access to water for 3 or $4 \mathrm{~h}$ before killing them. For thermal challenge, 2-month-old male PGC1 $\alpha$-null mice and WT littermates, or PGC1 $\alpha$-null mice infected with control or Ad-PGC1 $\alpha$ through tail vein injections, were anesthetized with isoflurane gas, immobilized and covered with a blanket and placed under a $250 \mathrm{~W}$ infrared lamp. Core rectal temperatures were monitored with a rectal thermometer (TH5, Braintree Scientific, Braintree, MA, USA) and raised to $41 \pm 0.5^{\circ} \mathrm{C}$ and kept at $41^{\circ} \mathrm{C}$ for $15 \mathrm{~min}$. After thermal challenge, mice were slowly returned to isothermal conditions over a warm blanket. Mice were killed and dissected $6 \mathrm{~h}$ after thermal challenge. The mice of the control group were also anesthetized with isoflurane gas, immobilized and covered with a blanket and monitored with a rectal thermometer in parallel, but maintained at room temperature for the same amount of time as the heat shock group.

Adenoviral delivery in inguinal fat and liver. Adenoviruses expressing control (CMV-GFP) and PGC1 $\alpha$ (mPGC1a-CMV-GFP) were constructed, amplified and purified by Vector Biolabs. Each of the adenoviruses $\left(5 \times 10^{9} \mathrm{pfu}\right)$ diluted in $50 \mu \mathrm{l}$ saline were injected into the inguinal fat pads of mice or $2 \times 10^{9} \mathrm{pfu}$ adenovirus through tail vein injection. Mice were killed at the forth day after viral delivery and tissues were dissected for gene analysis or for further heat shock treatment.

TUNEL assay and H\&E staining. WT, PGC1 $\alpha$-null cells and null cells with PGC1 $\alpha$ levels reconstituted were maintained in the incubator in a water bath at $37^{\circ} \mathrm{C}$ or $43^{\circ} \mathrm{C}$ for $1 \mathrm{~h}$ then exposed to a recovery time of 30 min at $37^{\circ} \mathrm{C}$. Cells were then fixed with fresh $4 \%$ paraformaldehyde and permeabilized with $0.1 \%$ Triton $\mathrm{X}-100$. Kidneys and livers from WT and PGC1 $\alpha \mathrm{KO}$ exposed to cold for $3 \mathrm{~h}$ and liver of PGC1 $\alpha$ KO mice infected with control or PGC1 $\alpha$ were dissected and tissues were fixed in $4 \%$ paraformaldehyde. Paraffin-embedded tissues were cut into sections of $5-\mu \mathrm{m}$ thickness. TUNEL assay (terminal deoxynucleotidyl transferasemediated dUTP-biotin nick end labeling) was performed to assess DNA fragmentation according to instructions provided by the manufacturer (Roche, 11 684817 910). Cells or tissue slides were analyzed using a microscope (Olympus, Tokyo, Japan) and images captured by a digital camera (Olympus) with 100- or 200fold magnification. Positive control was performed on a liver section treated with Dnase I prior to labeling procedure. To quantify TUNEL-positive cells, four individual wells or tissue slides from mice of each genotype were assayed. Ten fields were then randomly selected per well or slide. TUNEL-positive cells were counted on each field and averaged (percentage of positive cells per field was shown for cells and numbers of positive cells per field were shown for tissues). For toxicity analysis, BAT, liver, kidney and heart of mice injected with control or Ad-PGC1 $\alpha$ in iWAT were dissected and fixed in $4 \%$ paraformaldehyde and paraffin-embedded tissues were cut into section of $5-\mu \mathrm{m}$ thickness for $\mathrm{H} \& \mathrm{E}$ staining following standard procedures. ${ }^{21,23}$

Caspase-3 activity and cytosol cytochrome c (CytC) level measurement. Measurement of liver and kidney caspase-3 activity was performed using the Caspase-3 Colorimetric Assay Kit (Abcam, ab39401, Cambridge, UK) following the manufacturer's instructions. Livers and kidneys were cut into small pieces, washed three times with supplied cell lysis buffer and homogenized with a tissue grinder. Liver and kidney homogenates were centrifuged for $10 \mathrm{~min}$ at 12000 r.p.m. and supernatants were used for colorimetric determination at $405 \mathrm{~nm}$. The OD values were normalized to the protein levels present in the tissues. The homogenates were also used to isolate cytosolic fraction with Standard Cell Fractionation Kit (Abcam, ab109719) and cytosolic CytC levels were determined by Cytochrome c Profiling ELISA Kit (Abcam, ab110172) and normalized to protein levels present in the tissues.

Co-immunoprecipitation assays and western blots. Lysates from differentiated WT and PGC1 $\alpha$-null cells were incubated with anti-HSF1 antibody (Abgent, San Diego, CA, USA, AJ1374a) and rabbit IgG (Santa Cruz, Dallas, TX, USA, sc-2027) and performed on Catch and Release v2.0 reversible immunoprecipitation system following the manufacturer's instructions (Millipore, Billerica, MA, USA). The immune complexes were eluted and subjected to SDSPAGE. For immunoblot detection, we used anti-PGC1 $\alpha$ (Santa Cruz, sc-13067) and anti-HSF1 (Abgent AJ1374a antibodies). The secondary goat anti-rabbit antibody was purchased from Santa Cruz (sc-2030). For western blot, proteins were extracted from cells using RIPA buffer, consisting of $20 \mathrm{mM}$ Tris, $150 \mathrm{mM} \mathrm{NaCl}, 1 \%$ Triton X-100 and protease inhibitors (Roche) and separated in $4-20 \%$ or $10 \%$ Bis-Tris Gel (ThermoFisher, Rockville, MD, USA), transferred onto a PVDF membrane (Pierce, ThermoFisher) and incubated with primary antibodies including anti-Cleaved Caspase-3 (Cell Signaling, 9661, Danvers, MA, USA) and anti- $\beta$-actin (Sigma, A5316), overnight at $4^{\circ} \mathrm{C}$. Novex Sharp Pre-stained Protein Standard (ThermoFisher) was used as protein molecular size marker.

Chromatin immunoprecipitation assays. ChIP analyses of WT and PGC1 $\alpha$-null cells were performed using a ChIP assay kit (Millipore), following the manufacturer's recommendation and as previously described. ${ }^{21}$ Sonicated cell lysates were incubated with anti-IgG (Santa Cruz, sc-2027), anti-PGC1 $\alpha$ (Santa Cruz, sc-13067) or anti-HSF1 (Abgent, AJ1374a) antibodies for protein-DNA binding detection. The sequences of the primers used to assess the binding of HSF1 on the mouse Hsp70 promoter $(-272$ to +47) were the following: F: 5'- CAACAGTGTCAC TAGTAGCACC-3' and R: 5'- CTCTGGATGGAACCA GATTTGG-3' as previously described ${ }^{34}$ and on $\beta$-globin: F: 5'-AAGCCTGATTCCGTAGAGCCACAC-3' and R: 5'-CCCACAGGCAAGAGACAGCAGC-3'AAGCCTGATTCCGTAGAGCCACAC. ${ }^{21}$

Statistical analysis. Comparisons between groups were performed using $t$ test (GraphPad Software). Differences were considered significant with $P<0.05$. Error bars represent S.E.M. and results are shown as means \pm S.E.M.

\section{Conflict of Interest}

The authors declare no conflict of interest. 
Acknowledgements. We thank Pasha Sarraf for comments and are grateful to Bruce Spiegelman (Harvard Medical School) and Carl Wu (National Cancer Institute) for kindly providing reagents. This research was supported by funds of the NIDDK Intramural Research Program of the NIH.

1. Anckar J, Sistonen L. Regulation of HSF1 function in the heat stress response: implications in aging and disease. Annu Rev Biochem 2011; 80: 1089-1115.

2. Schlesinger MJ. Heat shock proteins. J Biol Chem 1990; 265: 12111-12114

3. Qiu XB, Shao YM, Miao S, Wang L. The diversity of the DnaJ/Hsp40 family, the crucial partners for Hsp70 chaperones. Cell Mol Life Sci 2006; 63: 2560-2570.

4. Young JC, Agashe VR, Siegers K, Hartl FU. Pathways of chaperone-mediated protein folding in the cytosol. Nat Rev Mol Cell Biol 2004; 5: 781-791.

5. Takayama S, Reed JC, Homma S. Heat-shock proteins as regulators of apoptosis. Oncogene 2003; 22: 9041-9047.

6. Verbeke P, Fonager J, Clark BF, Rattan SI. Heat shock response and ageing: mechanisms and applications. Cell Biol Int 2001; 25: 845-857.

7. Leak RK. Heat shock proteins in neurodegenerative disorders and aging. J Cell Commun Signal 2014; 8: 293-310.

8. McCarthy TV, Quane KA, Lynch PJ. Ryanodine receptor mutations in malignant hyperthermia and central core disease. Hum Mutat 2000; 15: 410-417.

9. Musselman ME, Saely S. Diagnosis and treatment of drug-induced hyperthermia. Am J Health Syst Pharm 2013; 70: 34-42.

10. Mayer MP, Bukau B. Hsp70 chaperones: Cellular functions and molecular mechanism. Cell Mol Life Sci 2005; 62: 670-684.

11. Neef DW, Jaeger AM, Thiele DJ. Heat shock transcription factor 1 as a therapeutic target in neurodegenerative diseases. Nat Rev Drug Discov 2011; 10: 930-944.

12. Xu YM, Huang DY, Chiu JF, Lau AT. Post-translational modification of human heat shock factors and their functions: a recent update by proteomic approach. J Proteome Res 2012; 11: 2625-2634

13. McMillan DR, Xiao X, Shao L, Graves K, Benjamin IJ. Targeted disruption of heat shock transcription factor 1 abolishes thermotolerance and protection against heat-inducible apoptosis. J Biol Chem 1998; 273: 7523-8528.

14. Xiao X, Zuo X, Davis AA, McMillan DR, Curry BB, Richardson JA et al. HSF1 is required for extra-embryonic development, postnatal growth and protection during inflammatory responses in mice. EMBO J 1999; 18: 5943-5952.

15. Vihervaara A, Sistonen L. HSF1 at a glance. J Cell Sci 2014; 127: 261-266.

16. Wu Z, Puigserver P, Andersson U, Zhang C, Adelmant G, Mootha V et al. Mechanisms controlling mitochondrial biogenesis and respiration through the thermogenic coactivator PGC-1 $\alpha$. Cell 1999; 98: 115-124.

17. Bagattin A, Hugendubler L, Mueller E. Transcriptional coactivator PGC-1 $\alpha$ promotes peroxisomal remodeling and biogenesis. Proc Natl Acad Sci U S A 2010; 107: 20376-20381.

18. St-Pierre J, Drori S, Uldry M, Silvaggi JM, Rhee J, Jäger $S$ et al. Suppression of reactive oxygen species and neurodegeneration by the PGC-1 $\alpha$ transcriptional coactivators. Cell 2006; 127: 397-408.

19. Lin J, Wu PH, Tarr PT, Lindenberg KS, St-Pierre J, Zhang CY et al. Defects in adaptive energy metabolism with CNS-linked hyperactivity in PGC-1 $\alpha$ null mice. Cell 2004; 119: 121-135.

20. Charos AE, Reed BD, Raha D, Szekely AM, Weissman SM, Snyder M. A highly integrated and complex PPARGC1A transcription factor binding network in HepG2 cells. Genome Res 2012; 22: 1668-1679.
21. Ma X, Xu L, Alberobello AT, Gavrilova O, Bagattin A, Skarulis M et al. Celastrol protects against obesity and metabolic dysfunction through activation of a HSF1-PGC1o transcriptional axis. Cell Metab 2015; 22: 695-708.

22. Puigserver P, Wu Z, Park CW, Graves R, Wright M, Spiegelman BM. A cold-inducible coactivator of nuclear receptors linked to adaptive thermogenesis. Cell 1998; 92 829-839.

23. Ma X, Xu L, Gavrilova O, Mueller E. Role of forkhead box protein $A 3$ in age-associated metabolic decline. Proc Natl Acad Sci U S A 2014; 111: 14289-14294.

24. Glazer JL. Management of heatstroke and heat exhaustion. Am Fam Physician 2005; 71 2133-2140.

25. Wu J, Ruas JL, Estall JL, Rasbach KA, Choi JH, Ye L et al. The unfolded protein response mediates adaptation to exercise in skeletal muscle through a PGC-1 $\alpha /$ ATF6 $\alpha$ complex. Cell Metab 2011; 13: 160-169.

26. Cullen KE, Sarge KD. Characterization of hypothermia-induced cellular stress response in mouse tissues. J Biol Chem 1997; 272: 1742-1746.

27. Štětina T, Koštál V, Korbelová J. The role of inducible Hsp70, and other heat shock proteins, in adaptive complex of cold tolerance of the fruit fly (Drosophila melanogaster). PLoS One 2015; 10: e0128976.

28. Chung J, Nguyen AK, Henstridge DC, Holmes AG, Chan MH, Mesa JL et al. HSP72 protects against obesity-induced insulin resistance. Proc Natl Acad Sci U S A 2008; 105 $1739-1744$.

29. Rera M, Bahadorani S, Cho J, Koehler CL, Ulgherait M, Hur JH et al. Modulation of longevity and tissue homeostasis by the Drosophila PGC-1 homolog. Cell Metab 2011; 14: 623-634.

30. Trinklein ND, Murray Jl, Hartman SJ, Botstein D, Myers RM. The role of heat shock transcription factor 1 in the genome-wide regulation of the mammalian heat shock response. Mol Biol Cell 2004; 15: 1254-1261.

31. Lee SH, Kwon HM, Kim YJ, Lee KM, Kim M, Yoon BW. Effects of hsp70.1 gene knockout on the mitochondrial apoptotic pathway after focal cerebral ischemia. Stroke 2004; 35 : 2195-2199.

32. Wacker JL, Huang SY, Steele AD, Aron R, Lotz GP, Nguyen Q et al. Loss of Hsp70 exacerbates pathogenesis but not levels of fibrillar aggregates in a mouse model of Huntington's disease. J Neurosci 2009; 29: 9104-9114.

33. Magnoni R, Palmfeldt J, Christensen JH, Sand M, Maltecca F, Corydon TJ et al. Late onset motoneuron disorder caused by mitochondrial Hsp60 chaperone deficiency in mice. Neurobiol Dis 2013; 54: 12-23.

34. Inouye S, Izu H, Takaki E, Suzuki H, Shirai M, Yokota Y et al. Impaired IgG production in mice deficient for heat shock transcription factor 1. J Biol Chem 2004; 279: 38701-38709.

(i)

Cell Death and Disease is an open-access journal published by Nature Publishing Group. This work is licensed under a Creative Commons Attribution 4.0 International License. The images or other third party material in this article are included in the article's Creative Commons license, unless indicated otherwise in the credit line; if the material is not included under the Creative Commons license, users will need to obtain permission from the license holder to reproduce the material. To view a copy of this license, visit http://creativecommons.org/licenses/by/4.0/ 